PROCEEDINGS OF THE

AMERICAN MATHEMATICAL SOCIETY

Volume 136, Number 7, July 2008, Pages 2535-2545

S 0002-9939(08)09366-0

Article electronically published on March 4, 2008

\title{
WHITNEY PROPERTY IN TWO DIMENSIONAL SOBOLEV SPACES
}

\author{
DORIN BUCUR, ALESSANDRO GIACOMINI, AND PAOLA TREBESCHI
}

(Communicated by Mario Bonk)

\begin{abstract}
For $p>1$, we prove that all the functions of $W_{\text {loc }}^{2, p}\left(\mathbb{R}^{2}\right)$ satisfy the Whitney property; i.e., if $u \in W_{\text {loc }}^{2, p}\left(\mathbb{R}^{2}\right)$ is such that $\nabla u=0$ (in the sense of capacity) on a connected set $K \subseteq \mathbb{R}^{2}$, then $u$ is constant on $K$.
\end{abstract}

\section{INTRODUCTION}

This paper deals with the Whitney property for Sobolev functions. In a regular context, a function $u \in C^{k}\left(\mathbb{R}^{N}\right), k \geq 1$, is said to have the Whitney property if the following holds: for every connected set $K \subseteq \mathbb{R}^{N}$ such that $\nabla u(x)=0$ for every $x \in K, u$ is constant on $K$.

The Whitney property is clearly implied by the assumption that the set of critical values of $u$ (namely the image of critical points) has zero measure. The problem of estimating the $M$-dimensional measure of the critical values of a function $u \in$ $C^{k}\left(\mathbb{R}^{N}, \mathbb{R}^{M}\right)$ is the main object of the so-called Morse-Sard Theorem: it turns out 20] that if $k>\max \{N-M, 0\}$, then the $M$-dimensional Lebesgue measure of the critical values of $u$ is zero. In the pioneering paper [22, Whitney constructed a function $u \in C^{1}\left(\mathbb{R}^{2}, \mathbb{R}\right)$ which is not constant on a connected set of critical points. This example can be generalized to an $N$-dimensional setting proving that the requirement $k>\max \{N-M, 0\}$ in the Morse-Sard Theorem is sharp.

The problem of weakening the differentiability assumption in the Morse-Sard theorem has been addressed in several papers: we refer the reader to the articles of Bates [4, 5] and of Norton [18, where the cases of $u \in C^{N-M}\left(\mathbb{R}^{N}, \mathbb{R}^{M}\right), N>M$, with $D^{N-M} u$ belonging to the family of Hölder continuous functions or to the Zygmund class are considered.

The Morse-Sard Theorem in the context of Sobolev spaces has been studied by De Pascale in [11] (see also [6] for some related results). He considers functions $u \in W_{\text {loc }}^{k, p}\left(\mathbb{R}^{N}, \mathbb{R}^{M}\right)$ with $N>M, k=N-M+1$ and $p>N$ : in this setting $u$ has a $C^{1}$ representative by the Sobolev Embedding Theorem, so that the set of critical values is classically defined, and he proves that it has zero $M$-dimensional measure. In the case $M=1$ and $N=2$, this result implies that a function $u \in W_{\text {loc }}^{2, p}\left(\mathbb{R}^{2}\right)$ with $p>2$ satisfies the Whitney property.

In this paper we discuss the Whitney property for Sobolev functions $u \in W_{\text {loc }}^{2, p}\left(\mathbb{R}^{2}\right)$ with $1<p \leq 2$. This situation is critical for De Pascale [11, and the Morse-Sard

Received by the editors May 15, 2007.

2000 Mathematics Subject Classification. Primary 46E35.

(C) 2008 American Mathematical Society Reverts to public domain 28 years from publication 2535 
theorem has an open issue (functions are not anymore $C^{1}$ ). Nevertheless, these functions admit continuous representatives, while their gradients, as elements of $W_{\text {loc }}^{1, p}\left(\mathbb{R}^{2}, \mathbb{R}^{2}\right)$, admit quasicontinuous representatives which are well defined up to sets with $c_{p}$-capacity zero (see Section 2 for the precise definitions). As a consequence, we consider sets $K$ which are contained in the set of critical points of $u \in W_{\text {loc }}^{2, p}\left(\mathbb{R}^{2}\right)$, up to a set of zero capacity, i.e. $\nabla u(x)=0$ for $c_{p}$-q.e. $x \in K$.

The main result of the paper consists in proving that all the functions of $W_{\mathrm{loc}}^{2, p}\left(\mathbb{R}^{2}\right)$ with $1<p \leq 2$ have the Whitney property in the following sense (see Theorem 4.3): if $K \subseteq \mathbb{R}^{2}$ is a connected set, and $u \in W_{\text {loc }}^{2, p}\left(\mathbb{R}^{2}\right)$ is such that $\nabla u=0 c_{p}$-q.e. on $K$, then $u$ is constant on $K$. In the case $p=2$ and for compact connected sets $K$, the result was stated (without proof) by Sverák in 21]. Our approach is based on methods from potential theory for Sobolev maps and on a duality argument which is proper of the two dimensional context.

First, we consider the case in which $K$ is bounded and connected, and $u \in$ $W^{2, p}\left(\mathbb{R}^{2}\right)$. Using capacity properties of connected sets in the plane, and using $p$ fine continuity properties of functions in $W^{1, p}$ (see Lemma 4.1), we prove that it is not restrictive to consider $K$ closed, i.e. compact. For some bounded open set $\Omega$ containing $K$, we prove that for every function $v \in W^{1,2}(\Omega \backslash K)$ we have (see Theorem 4.3 in Section 4)

$$
\int_{\Omega \backslash K} R \nabla u \cdot \nabla v d x=0
$$

where $R$ denotes a rotation of 90 degrees counterclockwise. The Whitney property follows since (1.1) turns out to imply (without any restriction for $\nabla u$ on $K$ ) the fact that $u$ is constant on $K$ (see Lemma 2.1). Notice that this certainly would be the case if $\partial K$ were smooth enough to perform an integration by parts. In fact, since $R \nabla u$ is divergence free, the left-hand side of (1.1) reduces to an integration of the tangential derivative $D_{\tau} u$ on $\partial \Omega \cup \partial K$ against (the trace of) $v$, and so $D_{\tau} u$ should vanish since $v$ is arbitrary. Here we prove that (1.1) implies that $u$ is constant on $K$ assuming only that $K$ is connected, and with no other geometric restrictions on its shape.

Second, the case of an arbitrary connected set $K$ is obtained by means of a slicing argument in polar coordinates, and employing classical Sard's theorem for the periodic one dimensional slicings in association with the first step. We emphasize at this point that the passage from bounded to unbounded sets $K$ is not of a topological nature, and we give an example of a continuous function which is constant on all bounded connected subsets of an unbounded connected set $K$, but which is not constant on the set $K$.

The paper is organized as follows. In Section 2 we recall some basic facts concerning capacity for Sobolev spaces and some results employed in the proofs of the main theorems. In Section 3 we derive some properties of connected sets in relation with capacity which are essential in the proofs of the main results contained in Section 4 .

\section{NOTATION AND PRELIMINARIES}

In this section we introduce the basic notation and recall some notions employed in the rest of the paper, which we give in a general $N$-dimensional setting even though our main result is two dimensional. 
If $\Omega \subseteq \mathbb{R}^{N}$ is open and $1 \leq p \leq+\infty$, we denote by $L^{p}(\Omega)$ the usual space of $p$-summable functions on $\Omega$ with norm indicated by $\|\cdot\|_{p} . W^{1, p}(\Omega)$ will denote the Sobolev space of functions in $L^{p}(\Omega)$ whose gradient in the sense of distributions belongs to $L^{p}\left(\Omega, \mathbb{R}^{N}\right)$, and we denote by $W_{0}^{1, p}(\Omega)$ the closure in $W^{1, p}(\Omega)$ of smooth functions with compact support in $\Omega$. With $W_{\text {loc }}^{1, p}\left(\mathbb{R}^{N}\right)$ we denote the space of function whose restriction to every relatively compact open set $\Omega \subseteq \mathbb{R}^{N}$ belongs to $W^{1, p}(\Omega)$.

If $E \subseteq \mathbb{R}^{N}$, we denote by $E^{c}$ its complementary set, and by $|E|$ its $N$-dimensional Lebesgue measure. For $x \in \mathbb{R}^{N}$ and $r>0$ we denote by $B_{x, r}$ the open ball with center $x$ and radius $r$. Finally, by a curve $\gamma$ connecting two points $x, \xi \in \mathbb{R}^{N}$ we mean a continuous function $\gamma:[a, b] \rightarrow \mathbb{R}^{N}$ such that $\gamma(a)=x$ and $\gamma(b)=\xi$ : we will denote by $\gamma_{[x, \xi]}$ the compact and connected image $\gamma([a, b])$.

Capacity. For convenience, we recall the definition of $c_{p}$-capacity we use in the estimates of the Wiener criterion, but we refer the reader to [15] for details concerning capacity, quasi and fine continuity. Let $\Omega$ be a bounded open set and $K$ a compact subset of $\Omega$. We set

$$
c_{p}(K, \Omega):=\inf \left\{\int_{\mathbb{R}^{N}}|\nabla \varphi|^{p}+|\varphi|^{p} d x: \varphi \in C_{c}^{\infty}(\Omega), \varphi \geq 1 \text { on } K\right\},
$$

and extend this definition to open and arbitrary sets by interior and exterior approximation, respectively. Properties holding quasi-everywhere (written $c_{p}$-q.e.) are properties which hold in the complement of a set of zero capacity (recall that if $E \subseteq \Omega \cap \Omega^{\prime}$, we have $c_{p}(E, \Omega)=0$ if and only if $c_{p}\left(E, \Omega^{\prime}\right)=0$, so that the notion of a set of zero capacity is independent of $\Omega$, and we can write simply $\left.c_{p}(E)=0\right)$.

Throughout the paper, we will identify a Sobolev function with its quasicontinuous representative. Notice that for $p>N$, the continuous representative of $u$ (which exists by the Sobolev Embedding Theorem) is precisely the quasicontinuous representative.

A representation result. We will use often the following representation result whose proof relies on the Helmholtz decomposition of $L^{2}$ (see [10, Lemma 3.6] or [13. Lemma 3.4]).

Lemma 2.1. Let $\Omega \subseteq \mathbb{R}^{2}$ be open and bounded, and let $q \geq 2$. Let $\psi \in L^{q}\left(\Omega, \mathbb{R}^{2}\right)$ be such that

$$
\int_{\Omega} \psi \cdot \nabla u d x=0 \quad \text { for every } u \in W^{1,2}(\Omega) .
$$

Then there exists $\phi \in W^{1, q}\left(\mathbb{R}^{2}\right)$ constant on the connected components of $\Omega^{c}$ (in the case $q=2$, constant $c_{2}$-quasi-everywhere) and such that

$$
\nabla \phi=R \psi \quad \text { in } \Omega
$$

where $R(a, b):=(-b, a)$ denotes a rotation of 90 degrees counterclockwise.

\section{Connected SETS AND CAPACITY}

In this section we briefly justify that a connected set $K$ in $\mathbb{R}^{N}$ is "large" in the sense of $c_{p}$-capacity; i.e., if $\operatorname{diam} K>0, x \in K$ and $0<r<\frac{1}{2} \operatorname{diam} K$ we have

$$
\frac{c_{p}\left(K \cap \bar{B}_{x, r}, B_{x, 2 r}\right)}{c_{p}\left(\bar{B}_{x, r}, B_{x, 2 r}\right)} \geq \frac{c_{p}\left([0,1] \times\{0\}^{N-1}, B_{0,2}\right)}{c_{p}\left(\bar{B}_{0,1}, B_{0,2}\right)} .
$$


Notice that the right-hand side of (3.1) is strictly positive for $p>N-1$, and this justifies the expression "large in the sense of $c_{p}$-capacity".

Inequality (3.1) was obtained in [8] in the case $N=2$ and $K$ compact, and extended in [9, Lemma 5.2] for arbitrary $N$. In this section we justify (3.1) for sets $K$ which are only connected. We use the following inequality (see 9]) concerning the capacity of curves. Let us consider a curve $\gamma_{[x, \xi]} \subseteq \bar{B}_{x, r}$ in $\mathbb{R}^{N}$ with extremes $x$ and $\xi$, such that $\xi \in \partial B_{x, r}$. Then

$$
c_{p}\left(\gamma_{[x, \xi]}, B_{x, 2 r}\right) \geq c_{p}\left([x, \xi], B_{x, 2 r}\right)
$$

where $[x, \xi]$ denotes the segment with extremes $x$ and $\xi$. The proof of this result relies on the Steiner symmetrization (see [7]).

Proposition 3.1. Let $K \subset \mathbb{R}^{N}$ be a connected set with $\operatorname{diam} K>0$. Then for all $x \in K$ and $0<r<\frac{1}{2} \operatorname{diam} K$ inequality (3.1) holds true.

Proof. Let $x \in K$. We have to evaluate $c_{p}\left(K \cap \bar{B}_{x, r}, B_{x, 2 r}\right)$. Recall that the capacity of $K \cap \bar{B}_{x, r}$ is the infimum of the capacity of all open sets in $B_{x, 2 r}$ which contain $K \cap \bar{B}_{x, r}$. Let $U \subseteq B_{x, 2 r}$ be such an open set, and let $U_{1}$ be the connected component of $U$ containing $x$. We claim that

$$
U_{1} \cap \partial B_{x, r} \neq \emptyset \text {. }
$$

In fact by contradiction, since $x \in U_{1}$ and $U_{1}$ is connected, we get $U_{1} \subseteq B_{x, r}$. As a consequence we have that

$$
\left(K \backslash \bar{B}_{x, r}\right) \cap \partial U_{1}=\emptyset .
$$

Clearly, since $U$ contains $K \cap \bar{B}_{x, r}$ and $\partial U_{1} \subseteq \partial U$, we have

$$
\left(K \cap \bar{B}_{x, r}\right) \cap \partial U_{1}=\emptyset,
$$

so that we can write

$$
K=\left(K \cap U_{1}\right) \cup\left(K \backslash \bar{U}_{1}\right),
$$

which contradicts the fact that $K$ is connected.

Taking into account (3.3), we can find $\xi_{r} \in U_{1}$ with $\left|\xi_{r}-x\right|=r$. Since $U_{1}$ is arcwise connected, there exists a continuous curve $\gamma_{\left[x, \xi_{r}\right]}$ contained in $U_{1}$ and joining $x$ and $\xi_{r}$. By Lemma 3.2 we deduce that

$c_{p}\left(U \cap \bar{B}_{x, r}, B_{x, 2 r}\right) \geq c_{p}\left(U_{1} \cap \bar{B}_{x, r}, B_{x, 2 r}\right) \geq c_{p}\left(\gamma_{\left[x, \xi_{r}\right]} \cap \bar{B}_{x, r}, B_{x, 2 r}\right) \geq c_{p}\left(\left[x, \xi_{r}\right], B_{x, 2 r}\right)$, where $\left[x, \xi_{r}\right]$ denotes the segment joining $x$ and $\xi_{r}$. We obtain

$$
\frac{c_{p}\left(U \cap \bar{B}_{x, r}, B_{x, 2 r}\right)}{c_{p}\left(\bar{B}_{x, r}, B_{x, 2 r}\right)} \geq \frac{c_{p}\left(\left[x, \xi_{r}\right], B_{x, 2 r}\right)}{c_{p}\left(\bar{B}_{x, r}, B_{x, 2 r}\right)} .
$$

Since

$$
\frac{c_{p}\left(\left[x, \xi_{r}\right], B_{x, 2 r}\right)}{c_{p}\left(\bar{B}_{x, r}, B_{x, 2 r}\right)}=\frac{c_{p}\left([0,1] \times\{0\}^{N-1}, B_{0,2}\right)}{c_{p}\left(\bar{B}_{0,1}, B_{0,2}\right)},
$$

the result follows by taking the infimum over $U$ in (3.4).

From Proposition 3.1 we get the Wiener-type formula (3.5) for $K$ : the limitations $N-1<p \leq N$ come from the fact that the result is trivial for $p>N$ since points have positive $c_{p}$-capacity, while inequality (3.1) (which we use to derive the result) gives no useful information for $p \leq N-1$ since segments have zero $c_{p}$-capacity. 
Proposition 3.2. Let $K \subseteq \mathbb{R}^{N}$ be a connected set with $\operatorname{diam} K>0, x \in K$ and let $N-1<p \leq N$. Then

$$
\int_{0}^{1}\left(\frac{c_{p}\left(K \cap B_{x, t}, B_{x, 2 t}\right)}{c_{p}\left(B_{x, t}, B_{x, 2 t}\right)}\right)^{\frac{1}{p-1}} \frac{d t}{t}=+\infty
$$

Proof. Let $\lambda \in] 0,1[$. Then we have the inequalities

$$
c_{p}\left(K \cap B_{x, t}, B_{x, 2 t}\right) \geq c_{p}\left(K \cap \overline{B_{x, \lambda t}}, B_{x, 2 t}\right) \geq C_{\lambda} c_{p}\left(K \cap \overline{B_{x, \lambda t}}, B_{x, 2 \lambda t}\right),
$$

where the last one comes by means of a rescaling argument for a suitable constant $C_{\lambda}>0$. Taking into account that $c_{p}\left(\bar{B}_{x, \lambda t}, B_{x, 2 \lambda t}\right)=c_{p}\left(B_{x, \lambda t}, B_{x, 2 \lambda t}\right)$, the result follows immediately from (3.1) since the right-hand side is strictly positive for $N-$ $1<p \leq N$.

In fact a connected set $K$ with positive diameter in $\mathbb{R}^{N}$ is uniformly $p$-thick for $N-1<p \leq N$ (see [15, Section 6.41]). A similar notion has been studied in [17] and is referred to as uniform fatness.

\section{The MAIn RESUlt}

In order to prove the Whitney property in the Sobolev space context $W_{\text {loc }}^{2, p}\left(\mathbb{R}^{2}\right)$, we need some preliminary lemmas.

Lemma 4.1. Let $K$ be a connected subset of $\mathbb{R}^{2}$. Let $1<p \leq 2$ and let $v \in$ $W_{\text {loc }}^{1, p}\left(\mathbb{R}^{2}\right)$ be such that $v=0 c_{p}$-q.e. on $K$. Then $v=0 c_{p}$-q.e. on $\bar{K}$.

Proof. We have to show that $v(x)=0$ for $c_{p}$-q.e. $x \in \bar{K} \backslash K$, where as usual we identify $v$ with its quasicontinuous representative. Since $v$ is quasicontinuous, by [16. Theorem 1.4] we have that $v$ is $p$-finely continuous up to a set of $c_{p}$-capacity zero. This means that there exists a set $A$ with $c_{p}(A)=0$ such that for every $x \in \mathbb{R}^{2} \backslash A$ there exists a set $E$ with

$$
\int_{0}^{1}\left(\frac{c_{p}\left(E \cap B_{x, t}, B_{x, 2 t}\right)}{c_{p}\left(B_{x, t}, B_{x, 2 t}\right)}\right)^{\frac{1}{p-1}} \frac{d t}{t}<+\infty
$$

such that (see [14, Theorem 3.17 and Corollary 3.18])

$$
v(x)=\lim _{y \rightarrow x, y \notin E} v(y) .
$$

Let us show that $v(x)=0$ for every $x \in \bar{K} \backslash(K \cup A)$. In view of (4.2), in order to get the conclusion it suffices to prove that there exists a sequence $y_{n} \in K \backslash(E \cup A)$ with $y_{n} \rightarrow x$, where $E$ is the exceptional set relative to $x$. By contradiction, let us assume that there exists $\varepsilon>0$ such that

$$
(K \backslash(E \cup A)) \cap B_{x, \varepsilon}=\emptyset
$$

so that

$$
K \cap B_{x, \varepsilon} \subseteq E \cup A .
$$

Since $K$ is connected, one can use Proposition 3.2 and since the critical behavior of the integral is near $t=0$, we obtain

$$
\int_{0}^{1}\left(\frac{c_{p}\left(\left(K \cap B_{x, \varepsilon}\right) \cap B_{x, t}, B_{x, 2 t}\right)}{c_{p}\left(B_{x, t}, B_{x, 2 t}\right)}\right)^{\frac{1}{p-1}} \frac{d t}{t}=+\infty .
$$


From (4.3), recalling that $c_{p}(A)=0$, we get that

$$
\int_{0}^{1}\left(\frac{c_{p}\left(E \cap B_{x, t}, B_{x, 2 t}\right)}{c_{p}\left(B_{x, t}, B_{x, 2 t}\right)}\right)^{\frac{1}{p-1}} \frac{d t}{t}=+\infty
$$

which means that $E$ does not satisfy (4.1), contradicting the assumption. The proof is thus concluded.

Notice that Lemma 4.1 has a natural extension to $\mathbb{R}^{N}$ provided that $N-1<$ $p \leq N$.

Lemma 4.2. Let $\Omega \subseteq \mathbb{R}^{2}$ be an open bounded connected set with Lipschitz boundary, and let $K \subseteq \Omega$ be compact. Let $1<p \leq 2$ and let $\Psi \in W_{0}^{1, p}\left(\Omega \backslash K, \mathbb{R}^{2}\right)$ with $\operatorname{div} \Psi=0$. Then for every $v \in W^{1,2}(\Omega \backslash K)$ we have

$$
\int_{\Omega \backslash K} \Psi \cdot \nabla v d x=0 .
$$

Proof. Notice that by the Sobolev Embedding Theorem we get that $\Psi=(A, B) \in$ $L^{p^{*}}\left(\Omega \backslash K, \mathbb{R}^{2}\right)$, where $p^{*}$ is the Sobolev exponent $p^{*}=\frac{2 p}{2-p}>2$, so that the integral in (4.4) is well defined (for $p=2, \Psi \in L^{q}\left(\Omega \backslash K, \mathbb{R}^{2}\right.$ ) for every $q<+\infty$ ). In order to prove (4.4), using a truncation argument, it is not restrictive to assume that

$$
v \in W^{1,2}(\Omega \backslash K) \cap L^{\infty}(\Omega \backslash K) .
$$

For every $n \in \mathbb{N}$ let us define

$$
K_{n}:=\left\{x \in \mathbb{R}^{2}: d(x, K) \leq \frac{1}{n}\right\}
$$

where $d(\cdot, K)$ denotes the distance function from $K$. Since $\Psi \in W_{0}^{1, p}\left(\Omega \backslash K, \mathbb{R}^{2}\right) \subseteq$ $W_{0}^{1, p}\left(\Omega, \mathbb{R}^{2}\right)$, we can find a sequence $\left(\Psi_{n}\right)_{n \in \mathbb{N}}$ such that $\Psi_{n} \in C_{c}^{\infty}\left(\Omega \backslash K_{n}, \mathbb{R}^{2}\right)$, and

$$
\Psi_{n} \rightarrow \Psi \quad \text { strongly in } W^{1, p}\left(\Omega, \mathbb{R}^{2}\right) .
$$

By the Sobolev Embedding Theorem we have that $\Psi_{n}$ is bounded in $L^{p^{*}}\left(\Omega, \mathbb{R}^{2}\right)$ (for $p=2, \Psi_{n}$ is bounded in $L^{q}\left(\Omega, \mathbb{R}^{2}\right)$ for every $q<+\infty$ ), and from (4.5) we get by interpolation that for every $p \leq r<p^{*}$ (for every $p \leq r<+\infty$ if $p=2$ )

$$
\Psi_{n} \rightarrow \Psi \quad \text { strongly in } L^{r}\left(\Omega, \mathbb{R}^{2}\right) .
$$

Let us fix $v \in W^{1,2}(\Omega \backslash K) \cap L^{\infty}(\Omega \backslash K)$. We get by (4.6) (choosing $2<r<p^{*}$ ) and integrating by parts

$$
\int_{\Omega \backslash K} \Psi \cdot \nabla v d x=\lim _{n \rightarrow+\infty} \int_{\Omega \backslash K_{n}} \Psi_{n} \cdot \nabla v d x=\lim _{n \rightarrow+\infty} \int_{\Omega \backslash K_{n}} \operatorname{div} \Psi_{n} \cdot v d x=0,
$$

since $\operatorname{div} \Psi_{n} \rightarrow \operatorname{div} \Psi=0$ strongly in $L^{p}(\Omega)$. We conclude that (4.4) holds, so that the proof is concluded.

Notice that Lemma 4.2 still holds for $p>2$ since $W^{1, p}(\Omega \backslash K)$ is contained in $W^{1,2}(\Omega \backslash K)$. We are now in a position to prove the main result of the paper.

Theorem 4.3. Let $K$ be a connected subset of $\mathbb{R}^{2}$, and let $u \in W_{\text {loc }}^{2, p}\left(\mathbb{R}^{2}\right)$ with $1<p \leq 2$ be such that $\nabla u=0 c_{p}$-q.e. on $K$. Then (the continuous representative of) $u$ is constant on $K$.

Proof. By Lemma 4.1 we can assume that $K$ is closed. We divide the proof into two steps. 
Step 1 ( $K$ is bounded). If we fix $\Omega$ to be an open, bounded and connected set in $\mathbb{R}^{2}$ such that $K \Subset \Omega$, upon multiplying $u$ by a smooth cut-off function, we may assume without restriction that $u=0$ outside $\Omega$. In particular we have that $u \in W^{2, p}(\Omega)$.

Let $R$ denote a rotation of 90 degrees counterclockwise. From the assumption we have that

$$
R \nabla u:=\left(g_{1}, g_{2}\right) \in W^{1, p}\left(\Omega, \mathbb{R}^{2}\right) \quad \text { with } \quad R \nabla u=0 c_{p} \text {-q.e. on } K,
$$

so that by [15, Theorem 4.5] (see also [3] )

$$
R \nabla u \in W_{0}^{1, p}\left(\Omega \backslash K, \mathbb{R}^{2}\right) .
$$

Moreover $R \nabla u$ has zero divergence. By Lemma 4.2 we get that for every $v \in$ $W^{1,2}(\Omega \backslash K)$,

$$
\int_{\Omega \backslash K} R \nabla u \cdot \nabla v d x=0 .
$$

By the Sobolev Embedding Theorem, we have that $R \nabla u \in L^{p *}\left(\Omega \backslash K, \mathbb{R}^{2}\right)(R \nabla u \in$ $L^{q}\left(\Omega \backslash K, \mathbb{R}^{2}\right)$ for every $q<+\infty$ if $\left.p=2\right)$, so that by Lemma 2.1 we get that there exists $\phi \in W^{1, p^{*}}\left(\mathbb{R}^{2}\right)$ with $\phi$ constant on $K$ such that

$$
R \nabla u=R \nabla \phi \quad \text { on } \Omega,
$$

so that

$$
u=\phi+\text { constant } \quad \text { on } \Omega .
$$

Since $\phi$ is constant on $K$, the conclusion follows, and Step 1 is concluded.

Step 2 ( $K$ is unbounded). Let us employ polar coordinates $(r, \vartheta)$, i.e., let us identify a point in $\mathbb{R}^{2}$ with the complex number $r e^{i \vartheta}$. It is not restrictive to assume that $0 \in K$. Since $K$ is unbounded, for every $r>0$ we have that $K \cap \partial B_{r}(0) \neq \emptyset$ : let us set

$$
K_{r}:=\left\{\vartheta \in[0,2 \pi]: r e^{i \vartheta} \in K\right\} .
$$

We claim that we can find a sequence $r_{n} \nearrow+\infty$ with

$$
u(K)=\bigcup_{n \in \mathbb{N}} u\left(K \cap \partial B_{r_{n}}(0)\right),
$$

and such that setting $u_{n}(\vartheta):=u\left(r_{n} e^{i \vartheta}\right)$ we have

$$
u_{n} \in W^{2, p}\left(S^{1}\right)
$$

Then the conclusion follows by the absolute continuity of $u_{n}$, which in view of (4.10) implies that $\mathcal{L}^{1}\left(u_{n}\left(K_{r_{n}}\right)\right)=0$ for every $n \in \mathbb{N}$. In fact by (4.8) we deduce that

$$
\mathcal{L}^{1}(u(K)) \leq \sum_{n=0}^{+\infty} \mathcal{L}^{1}\left(u_{n}\left(K_{r_{n}}\right)\right)=0 .
$$

Since $u(K)$ is an interval, we get that $u(K)=\{c\}$ for some $c \in \mathbb{R}$, and the Whitney property follows.

In order to conclude the proof, we need to prove claims (4.8), (4.9) and (4.10). Let us start considering (4.8). Let $x \in K$, and let $r>0$ be such that $x \in B_{r}(0)$. Let us consider for $\delta>0$,

$$
K^{\delta}:=\left\{x \in \mathbb{R}^{2}: d(x, K)<\delta\right\} .
$$


We have that $K^{\delta}$ is open and connected (since $K$ is connected), and since $K$ is unbounded, there exists $\xi_{r}^{\delta} \in K^{\delta} \cap \partial B_{r}(0)$. Let $\gamma_{r}^{\delta}$ be a curve in $K^{\delta}$ connecting $x$ and $\xi_{r}^{\delta}$ : up to replacing $\xi_{r}^{\delta}$ with the first point in which $\gamma_{r}^{\delta}$ intersects $\partial B_{r}(0)$, we can assume that $\gamma_{r,\left[x, \xi_{r}^{\delta}\right]}^{\delta} \subseteq K^{\delta} \cap \bar{B}_{r}(0)$. As $\delta \rightarrow 0$, we have that up to a subsequence,

$$
\gamma_{r,\left[x, \xi_{r}^{\delta}\right]}^{\delta} \rightarrow \gamma_{r} \quad \text { in the Hausdorff metric, }
$$

where $\gamma_{r} \subseteq K \cap \bar{B}_{r}(0)$ is a compact and connected set such that $x, \xi_{r} \in \gamma_{r}$ for some $\xi_{r} \in K \cap \partial B_{r}(0)$ (for the details concerning the Hausdorff metric we refer the reader to [19]). Since $\nabla u=0 c_{p}$-q.e. on $\gamma_{r}$, by Step 1 we get that $u$ is constant on $\gamma_{r}$. In particular $u(x)=u\left(\xi_{r}\right)$. In other words, we have proved that the value of $u$ at $x$ can be recovered from the values of $u$ on $\partial B_{r}(0)$. Thus we conclude that for every $r_{n} \nearrow+\infty$ equality (4.8) holds.

Claims (4.9) and (4.10) follow by the standard theory of slicing properties of Sobolev functions (see e.g. [12, Section 4.9]): for a.e. $\bar{r}>0$ we have that the function $u_{\bar{r}}(\vartheta):=u\left(\bar{r} e^{i \vartheta}\right)$ belongs to $W^{2, p}\left(S^{1}\right)$ and is such that for a.e. $\vartheta \in[0,2 \pi]$,

$$
u_{\bar{r}}^{\prime}(\vartheta)=\nabla u\left(\bar{r} e^{i \vartheta}\right) \cdot \frac{e_{\vartheta}}{\bar{r}},
$$

where $e_{\vartheta}:=(-\sin \vartheta, \cos \vartheta)$. Recall that by [12, Section 4.7.2, Theorem 4] we have that for every $E \subseteq \mathbb{R}^{2}$ and $q \geq 1$,

$$
c_{q}(E)=0 \Longrightarrow \mathcal{H}^{s}(E)=0 \quad \text { for every } s>2-q .
$$

In particular for $1<p \leq 2$,

$$
c_{p}(E)=0 \Longrightarrow \mathcal{H}^{1}(E)=0 .
$$

Since $\nabla u=0 c_{p}$-q.e. on $K$, by (4.11) and (4.12) we have that $u_{\bar{r}}^{\prime}(\vartheta)=0$ for a.e. $\vartheta \in K_{\bar{r}}$. Then claims (4.9) and (4.10) are proved, and the proof is concluded.

Remark 4.4. The proof of Step 1 of Theorem 4.3, which is based on Lemmas 4.2 and 2.1, can be summarized as follows.

(1) We consider the vector field $g:=R \nabla u$, which is divergence free and vanishes $c_{p}$-q.e. on $K$.

(2) We approximate $g$ (in the $L^{2}$-norm) by smooth divergence free vector fields $\left(g_{n}\right)_{n \in \mathbb{N}}$ vanishing on neighborhoods of $K$.

(3) From $g_{n}$ we construct a sequence of functions $\left(u_{n}\right)_{n \in \mathbb{N}}$ such that $u_{n}$ converges in $W^{1, p}$ to $u$ and $u_{n}$ is constant on $K$ (since $\left.\nabla u_{n}=R^{-1} g_{n}\right)$. As a consequence, $u$ turns out to be constant on $K$.

Clearly point (1), that is, associating a divergence free vector field to $\nabla u$, can be carried out easily in even dimensions. Also the approximation of point (2) holds in higher dimensions since it is based on the Helmholtz decomposition of $L^{2}$. Point (3) is however essentially two dimensional, and we do not know any operation similar to $R^{-1}$ which associates, in higher dimensions, the gradient of a function to a divergence free vector field.

Remark 4.5. Under the assumptions of Theorem 4.3, the result of De Pascale [1] cannot be applied since $1<p \leq 2$. Moreover, the proof of our result relies on arguments strictly connected to the notion of the $p$-fine topology, which are different from those used in [11. 
Remark 4.6. The key point in the proof of Step 2 of Theorem 4.3 is the use of the absolute continuity for the regular one dimensional sections of $u$ which gives nontrivial information on $u(K)$ in view of (4.8). A decomposition with countably many sections such as in (4.8) still holds if $K$ is no more connected but has a countable number of connected components, but it clearly fails in the general case.

Remark 4.7. Notice that in Step 2 of the proof of Theorem 4.3 we used the fact that $u \in W_{\text {loc }}^{2, p}\left(\mathbb{R}^{2}\right)$ to carry over a dimensional reduction through slicing in polar coordinates and we used absolute continuity on the countably many sections needed (see (4.9) and (4.10) ). Thus to get the conclusion for the case that $K$ is unbounded we needed an "energetic" argument (the Sobolev regularity) together with the Whitney property for bounded connected sets (which we proved in Step 1). We remark that we cannot get the conclusion using simply topological arguments, i.e., using only Step 1 and the continuity of $u$. In fact we can consider the following example.

Let $C:=[0,1] \backslash \bigcup_{n=1}^{\infty} E_{n}$ be the usual Cantor set contained in $[0,1]$, where $E_{n}$ is the union of the middle-third intervals, i.e.,

$$
\left.E_{1}=\right] 1 / 3,2 / 3\left[, \quad E_{2}=\right] 1 / 9,2 / 9[\cup] 7 / 9,8 / 9[\quad \ldots
$$

and so on. Let $E_{n}:=\bigcup_{k=1}^{k_{n}} I_{k}^{n}$ with $\left.I_{k}^{n}=\right] a_{k}^{n}, b_{k}^{n}$. For every $k$, let $\gamma_{k}^{n}: I_{k}^{n} \rightarrow \mathbb{R}$ be a continuous function such that

$$
\liminf _{x \rightarrow a_{k}^{n}} \gamma_{k}^{n}(x)=-\infty, \quad \limsup _{x \rightarrow a_{k}^{n}} \gamma_{k}^{n}(x)=+\infty,
$$

and

$$
\liminf _{x \rightarrow b_{k}^{n}} \gamma_{k}^{n}(x)=-\infty, \quad \limsup _{x \rightarrow b_{k}^{n}} \gamma_{k}^{n}(x)=+\infty .
$$

Let $\Gamma_{k}^{n} \subseteq I_{k}^{n} \times \mathbb{R}$ be the image of $\gamma_{k}^{n}$. For every $n \in \mathbb{N}$ let us set

$$
H^{n}:=\bigcup_{k=1}^{k_{n}} \Gamma_{k}^{n},
$$

and let

$$
K:=(C \times \mathbb{R}) \cup \bigcup_{n \in \mathbb{N}} H^{n} .
$$

We have that $K \subseteq \mathbb{R}^{2}$ is closed and connected. Let us consider $u(x, y)=f(x)$, where $f$ is the usual Cantor-Vitali function (see e.g. [2, Example 1.67]). The function $u$ (which has bounded variation (see [2]) but does not belong to $W_{\text {loc }}^{2, p}$ ) is constant on every bounded and connected set $H \subseteq K$, but it is not globally constant on $K$.

Remark 4.8. We conclude the section by noticing an extension of a result proved by Sverák 21] (see Lemma 5.2 and the Remark on page 548) in the case $p=2$ : the proof relies on Lemma 4.2

Let $\Omega$ be an open bounded set in $\mathbb{R}^{2}$ such that the number of connected components of $\mathbb{R}^{2} \backslash \Omega$ is finite. Then for every $\left.p \in\right] 1,+\infty[$ the space

$$
V(\Omega):=\left\{\Psi \in W_{0}^{1, p}\left(\Omega, \mathbb{R}^{2}\right): \operatorname{div} \Psi=0\right\}
$$

coincides with the closure in $W_{0}^{1, p}\left(\Omega, \mathbb{R}^{2}\right)$ of

$$
\mathcal{V}(\Omega):=\left\{\Psi \in C_{c}^{\infty}\left(\Omega, \mathbb{R}^{2}\right): \operatorname{div} \Psi=0\right\} .
$$


In order to prove this result, it suffices to check that every $\Psi \in V(\Omega)$ is a limit in the topology of $W^{1, p}\left(\Omega, \mathbb{R}^{2}\right)$ of elements of $\mathcal{V}(\Omega)$. Let $B$ be an open ball such that $\bar{\Omega} \subseteq B$, and let us set $K:=\bar{B} \backslash \Omega$. We have that $K$ has a finite number of connected components.

Let us consider the case $1<p<2$. By Lemma 4.2 we get for every $v \in$ $W^{1,2}(B \backslash K)$,

$$
\int_{B \backslash K} \Psi \cdot \nabla v d x=0
$$

Notice that by the Sobolev Embedding Theorem we have $\Psi \in L^{p^{*}}\left(B \backslash K, \mathbb{R}^{2}\right)$, where $p^{*}$ is the Sobolev exponent $p^{*}=\frac{2 p}{2-p}>2$. By Lemma 2.1 we deduce that there exists $\phi \in W^{1, p^{*}}\left(\mathbb{R}^{2}\right)$ constant on the connected components of $K$ such that

$$
\Psi=R \nabla \phi \quad \text { on } B \backslash K,
$$

where $R$ denotes a rotation of 90 degrees counterclockwise. We get that $\phi \in$ $W^{2, p}\left(\mathbb{R}^{2}\right)$. Since $K$ has a finite number of connected components, we can find a function $h \in C_{c}^{\infty}(B)$ whose gradient vanishes in a neighborhood of $K$ and such that it coincides with $\phi$ on $K$. Let us consider the function $\theta:=\phi-h \in W_{0}^{2, p}(B \backslash K)$. Then, by [1, Theorem 9.1.3], there exists $\theta_{j} \in C_{c}^{\infty}(B \backslash K)$ such that

$$
\theta_{j} \rightarrow \theta \quad \text { strongly in } W_{0}^{2, p}(B \backslash K) .
$$

Let us define the functions

$$
\Psi_{j}=R \nabla \theta_{j}+R \nabla h .
$$

Recalling that $B \backslash K=\Omega$, by construction we have $\Psi_{j} \in C_{c}^{\infty}\left(\Omega, \mathbb{R}^{2}\right)$, $\operatorname{div} \Psi_{j}=0$ and

$$
\Psi_{j} \rightarrow R \nabla \phi=\Psi \quad \text { strongly in } W_{0}^{1, p}\left(\Omega, \mathbb{R}^{2}\right),
$$

so that the result follows.

The case $p \geq 2$ follows the same lines as the case $1<p<2$ : it suffices to take into account that by the Sobolev Embedding Theorem, $\Psi$ is either an $L^{q}$-function for every $1 \leq q<+\infty$ or a Hölder continuous function so that the representation formula (4.13) with $\phi \in W^{2, p}\left(\mathbb{R}^{2}\right)$ still holds.

\section{REFERENCES}

1. Adams, D. R., Hedberg, L. I.: Function spaces and potential theory, Grundlehren der Mathematischen Wissenschaften [Fundamental Principles of Mathematical Sciences], 314. SpringerVerlag, Berlin, 1996. MR.1411441 (97j:46024)

2. Ambrosio, L., Fusco, N., Pallara, D.: Functions of bounded variations and free discontinuity problems. Clarendon Press, Oxford, 2000. MR1857292 (2003a:49002)

3. Bagby, T.: Quasi topologies and rational approximation. J. Funct. Anal. 10 (1972), 259-268. MR0355058 (50:7535)

4. Bates, S. M.: On the image size of singular maps. I. Proc. Amer. Math. Soc. 114, 3 (1992), 699-705. MR1074748 (92f:58015)

5. Bates, S. M.: On the image size of singular maps. II. Duke Math. J. 68, 3 (1992), 463-476. MR.1194950 (94e:58009)

6. Bojarski, B., Hajłasz, P., Strzelecki, P.: Sard's theorem for mappings in Hölder and Sobolev spaces. Manuscripta Math. 118 (2005), no. 3, 383-397. MR2183045 (2007b:58014)

7. Brock, F.: Continuous rearrangement and symmetry of solutions of elliptic problems. Proc. Indian Acad. Sci. Math. Sci. 110, no. 2, (2000), 157-204. MR1758811(2001i:35016)

8. Bucur, D.: Shape analysis for nonsmooth elliptic operators. Appl. Math. Lett. 9 (1996), no. 3, 11-16. MR1385991 (96m:35062) 
9. Bucur, D., Trebeschi, P.: Shape optimisation problems governed by nonlinear state equations. Proc. Roy. Soc. Edinburgh Sect. A 128 (1998), 945-963. MR1642112 (99i:49048)

10. Dal Maso, G., Ebobisse, F., Ponsiglione, M.: A stability result for nonlinear Neumann problems under boundary variations. J. Math. Pures Appl. 82 (2003), 503-532. MR1995490 (2005b:35092)

11. De Pascale, L.: The Morse-Sard theorem in Sobolev spaces. Indiana Univ. Math. J. 50 (2001), 1371-1386. MR1871360 (2002k:58018)

12. Evans, L. C., Gariepy, R. F.: Measure Theory and Fine Properties of Functions, CRC Press, Boca Raton, FL, 1992. MR1158660 (93f:28001)

13. Giacomini, A., Trebeschi, P.: A density result for Sobolev spaces in dimension two, and applications to stability of nonlinear Neumann problems. J. Differential Equations 237 (2007), 27-60. MR 2327726

14. Heinonen, J., Kilpelainen, T., Martio, O.: Fine topology and quasilinear elliptic equations. Annales de l'Institut Fourier 39, 2 (1989), 293-318. MR:1017281 (91b:31015)

15. Heinonen, J., Kilpeläinen, T., Martio, O.: Nonlinear potential theory of degenerate elliptic equations. Oxford Mathematical Monographs. Oxford Science Publications. The Clarendon Press, Oxford University Press, New York, 1993. MR.1207810 (94e:31003)

16. Kilpeläinen, T., Malý, J.: Supersolutions to degenerate elliptic equation on quasi open sets. Comm. Partial Differential Equations 17, 3-4 (1992), 371-405. MR.1163430 (93g:31022)

17. Lewis, J.: Uniformly fat sets. Trans. Amer. Math. Soc. 308 (1988), no. 1, 177-196. MR946438 (89e:31012)

18. Norton, A.: A critical set with nonnull image has large Hausdorff dimension. Trans. Amer. Math. Soc. 296, no. 1 (1986), 367-376. MR837817 (87i:26011)

19. Rogers, C. A.: Hausdorff Measures. Cambridge University Press, Cambridge, 1970. MR 0281862 (43:7576)

20. Sard, A.: The measure of the critical values of differentiable maps. Bull. Amer. Math. Soc. 48 (1942), 883-890. MR0007523 (4:153c)

21. Sverák, V.: On optimal shape design. J. Math. Pures Appl. 72 (1993), 537-551. MR1249408 $(94 \mathrm{j}: 49047)$

22. Whitney, H.: A function not constant on a connected set of critical points. Duke Math. J. 1 (1935), 514-517. MR 1545896

Laboratoire de Mathématiques, CNRS UMR 5127 Université de Savoie, Campus Scientifique, 73376 Le-Bourget-Du-Lac, France

E-mail address: dorin.bucur@univ-savoie.fr

Dipartimento di Matematica, Facoltà di Ingegneria, Università degli Studi di BresCia, Via Valotti 9, 25133 Brescia, Italy

E-mail address: alessandro.giacomini@ing.unibs.it

Dipartimento di Matematica, Facoltà di Ingegneria, Università degli Studi di BresCia, Via Valotti 9, 25133 Brescia, Italy

E-mail address: paola.trebeschi@ing.unibs.it 